eine ‘ökologische Steuerreform’ eine wesentliche Rolle spielen (4). Soweit durch eine solche Reform die bürokratische Umweltpolitik, wenn auch nicht ganz abgelöst, so doch in den Hintergrund gedrängt wird, führt sie zu Effizienzgewinnen. Da die Erhöhung der Produktionskosten durch die Verteuerung der Energie durch die Verringerung der Arbeitskosten zumindest teilweise kompensiert wird, ist eine solche Politik auch angesichts der wirtschaftlichen Globalisierung möglich.

Dies aber bedeutet, daß weder die wirtschaftliche Globalisierung nationale Umweltpolitik verunmöglicht, noch globale Umweltprobleme ohne politische Antwort bleiben müssen. In beiden Fällen wird die Umweltpolitik schwieriger und erfordert deshalb mehr (politische) Phantasie als zuvor, aber es stehen dennoch auch nationale umweltpolitische Handlungsmöglichkeiten zur Verfügung. Diese sollten gerade dann offensiv genutzt werden, wenn sich die Erzielung wirksamer internationaler Abkommen als schwierig erweist.

\section{Anmerkungen}

(1) Kirchgässner, G.: Probleme internationaler Umweltpolitik, in: E. Gawel (Hg.): Institutionelle Probleme der Umweltpolitik, Sonderheft 8/1996 der Zeitschrift für Angewandte Umwelfforschung, S.157-172.

(2) $\mathrm{Vgl}$. zu den Möglichkeiten eines kleinen Landes wie der Schweiz: Jochimsen, M., G.Kirchgüssner (Hg.): Schweizerische Umweltpolitik im internationalen Kontext, Birkhäuser, Bosel 1995.

(3) Vgl. z.B. Stieger, R.: Internationaler Umweltschutz: Eine politisch-ökonomische Analyse der Verträge zum Schutz der Ozonschicht, Peter Lang, Bern u.a. 1995.

(4) Zu Einzelheiten vgl. Kirchgässner, G.: Ökologische Steuerreform: Utopie oder realistische Alternative?, in: G. Krause-Junk (Hg.): Die Zukunft des Steuersystems, Sonderheft der Zeitschrift für Wirtschafts- und Sozialwissenschaften, Duncker und Humblot, Berlin 1998, S.279-319

\section{Der Autor}

Dr. Gebhard Kirchgässner ist Professor für Volkswirtschaftslehre und Ökonometrie sowie Direktor des Schweizerischen Instituts für AußenwirtschaftsStruktur- und Regionalforschung an der Universität Si. Gallen.

Kontakt: Universität Si. Gallen, SIASR, Institutsgebäude, Dufourstr. 48, CH-9000 St.Gallen. Tel: $++41 / 71 / 224-2347$.

E-mail: Gebhard.Kirchgaessner@SIASR.unisg.ch

Die europäische Integration im Vergleich mit globalen Entwicklungen

\title{
Liberalisierung und Umweltschutz
}

\author{
Im Zusammenhang mit der Globalisierung von Märkten wird vielfach die \\ Befürchtung eines umweltpolitischen "race to the bottom "geäußert. Der \\ europäische Fall zeigt jedoch, daß ökonomische Integration und Umweltschutz \\ keineswegs unvereinbare Ziele sein müssen, sofern eine geeignete Dynamik \\ supranationaler Kooperation entsteht.
}

$\mathrm{H}$ Von Christoph Knill äufig wird angenommen, daß bei zunehmender ökonomischer Interdependenz strenge Umweltstandards die nationale Industrie belasten und damit deren internationale Wettbewerbsfähigkeit unnötig schwächen würden. Vergleicht man dieses Szenario jedoch mit der Entwicklung der Umweltpolitik im Rahmen der Europäischen Union (EU), so scheint diese Prognose keineswegs universale Gültigkeit zu haben. Die Verwirklichung des europäischen Binnenmarktes hat nicht zu einem umweltpolitischen "race to the bottom" geführt. Vielmehr haben sich nationale Umweltregulierungen unter europäischem Einfluß tendenziell weiter verschärft (1). Es ist an dieser Stelle zu betonen, daß die Einschätzung der europäischen Umweltpolitik relativ zu globalen Entwicklungen erfolgt. Genauer betrachtet ist das Bild natürlich keineswegs so makellos, wie es sich aus dieser, ,Vogelperspektive" darstellt, nimmt man etwa die Vollzugsdefizite europäischer Umweltpolitik als Beispiel (2). Vor dem Hintergrund dieser relativ erfolgreichen Symbiose von Marktliberalisierung und Umweltschutz stellt sich die Frage, welche Bedingungen die europäische Entwicklung begünstigt haben und inwieweit diese Faktoren auch im Hinblick auf globale Umweltregulierung gegeben sind.

Betrachtet man die Entwicklung der europäischen Umweltpolitik, so fallen mehrere Aspekte auf, die auf den ersten Blick relativ überraschend sind - zumindest aus der Sicht der eingangs vorgestellten Annahme eines umweltpolitischen Dumpings. Erstens läßt sich eine graduelle Emanzipation der Umweltpolitik als eigenständiges Handlungsfeld der Gemeinschaft beobachten; d.h. Umweltpolitik wird nicht als Anhängsel, sondern losgelöst von Fragen der wirtschaftlichen Integration betrachtet (3). Diese Entwicklung wurde rechtlich bestätigt durch die vertragliche Verankerung von Umweltpolitik als eigenständiges Handlungsfeld der Gemeinschaft im Rahmen der Einheitlichen Europäischen Akte 1986 und deren Forfführung im Vertrag von Maastricht. Ein zweiter Aspekt, welcher die Entwicklung der europäischen Umweltpolitik charakterisiert, ist die relative, wenngleich unstetig verlaufende, Verschärfung umweltpolitischer Vorgaben. Diese bezieht sich nicht nur auf materielle Regulierungen (z.B. Emissionsgrenzwerte), sondern auch auf prozedurale Vorgaben (wie etwa den freien Zugang zu Umweltinformationen). Drittens ist schließlich die breite Mischung von verschiedenen Regulierungsmustern zu erwähnen. Europäische Umweltpolitik besteht keineswegs aus einem einheitlichen regulativen Konzept, sondern umfaßt eine Mischung von regulativen Ansätzen, welche von materiellen Standards und prozeduralen Vorgaben bis hin zur Selbstregulierung der Industrie reicht. Fragt man nach den Bedingungen, welche diese Entwicklung begünstigt haben, so lassen sich ökonomische und politische Mechanismen unterscheiden.

\section{Umwelidynamik des Binnenmarktes}

Wie David Vogel kürzlich gezeigt hat, muß ökonomische Integration keineswegs ein umweltpolitisches ,race to the bottom“ zur Folge haben, sondern kann unter bestimmten Bedingungen sogar ein „race to the top" begünstigen, d.h. eine Verschärfung von umweltpolitischen Regulierungen (4).

Einerseits ist es insbesondere in umweltpolitisch hochentwickelten Staaten denkbar, daß die Industrie für eine weitere Verschärfung nationaler Umweltstandards eintritt, um Wettbewerbsvorteile gegenüber ausländischen Konkurrenten zu erlangen. Dies ist dann der Fall, wenn die nationale Industrie bereits auf solche Standards einge- 
stellt ist und damit kostengünstiger und schneller als die ausländische Konkurrenz den nationalen Markt bedienen kann. In solchen Situationen kommt es vielfach zu einer Interessenkongruenz von nationaler Industrie und Umweltverbänden, was das politische Gewicht solcher Forderungen erhöht.

Auf der anderen Seite besteht die Möglichkeit, daß von strengen Standards in ,grünen“ Staaten ein Pull-Effekt auf weniger streng regulierte ausländische Märkte ausgeht. Dies ist dann zu erwarten, wenn dort Industrien ansässig sind, die in starkem Maße von Exporten in diese ,grünen“ Märkte abhängen.

Den regulierungsverstärkenden Effekten, die sich aus der Binnenmarktdynamik ergeben, sind allerdings gewisse Grenzen gesetzt. Die erste Grenze ist rechtlicher Natur.

Die Verschärfung nationaler Umweltstandards darf nicht zu einer Beeinträchtigung des freien Warenverkehrs im Rahmen liberalisierter Märkte fuihren. Mit anderen Worten, umweltpolitische Maßnahmen sind dann unzulässig, wenn sie als sog. „nicht-tarifäres Handelshemmnis“ klassifiziert werden. Es muß stets nachgewiesen werden, daß entsprechende Maßnahmen primär dem Schutz der Umwelt dienen und nicht dem Schutz nationaler Märkte. In der Praxis stellt sich die Beurteilung vielfach als Balanceakt dar, der nicht selten vom Europäischen Gerichtshof entschieden wurde.

Zweitens muß betont werden, daß die umweltschutzverstärkenden Effekte des Binnenmarktes sich grundsätzlich auf die Regulierung von Produktstandards beschränken. Produktstandards beziehen sich auf Vorgaben über die Beschaffenheit bestimmter Güter, zum Beispiel Abgaswerte von Autos oder den Schwefelgehalt von Brennstoffen. Die umweltpolitische Dynamik des Binnenmarktes kommt hingegen weniger zur Geltung, wenn es sich um sog. Produktionsstandards handelt. Letztere beziehen sich auf die Bedingungen, unter denen bestimmte Güter hergestellt werden, wie etwa den Umfang industrieller Emissionen, die im Produktionsprozeß anfallen. Da Produktionsstandards unmittelbar die Produktionskosten und damit die wirtschaftliche Wettbewerbsfähigkeit von Ländern beeinflussen, müßte eine verstärkte ökonomische Integration daher hier eher ein umweltpolitisches ,,race to the bottom" als ein „race to the top“ in Gang setzen. Insgesamt sind die Effekte der Marktintegration im Hinblick auf strengere umweltpolitische Regelungen relativ eingeschränkt. Rein ökono- mische Mechanismen reichen also nicht aus, um die eingangs geschilderte Entwicklung der europäischen Umweltpolitik zu erklären.

\section{- Dynamik supranationaler politischer Kooperation}

Die Tatsache, daß die Umweltpolitik der EU nicht nur im Hinblick auf Produktstandards, sondern auch beziiglich der Regulierung von Produktionsstandards wichtige Fortschritte gemacht hat, läßt sich daher nur begreifen, wenn man die Dynamik politischer Kooperation im Rahmen der EU berïcksichtigt. Diese Dynamik entspringt zwei Faktoren: dem supranationalen Charakter der Gemeinschaft und spezifischen Merkmalen ihrer Mitgliederstruktur.

Der supranationale Charakter der EU, der sich daraus ergibt, daß die Mitgliedsstaaten einen Teil ihrer Kompetenzen auf europäische Institutionen übertragen haben, ermöglicht eine dynamische Entwicklung der europäischen Umweltpolitik aufgrund zweier spezifischer Begleiterscheinungen: dem institutionellen Kompetenzwettbewerb zwischen supranationalen Institutionen und Nationalstaaten sowie dem regulativen Wettbewerb zwischen den Mitgliedsstaaten. Beide Elemente erlauben umweltpolitisch ambitionierten Vorreitern potentiell, ihre Standards auch fuir andere Länder verbindlich zu machen. Institutioneller Wettbewerb bedeutet, daß die supranationalen Organe wie Kommission und Europäisches Parlament grundsätzlich bestrebt sind, ihre Kompetenzen und Handlungsspielräume gegenüber den Mitgliedsstaaten zu erweitern. Ein wichtiges Mittel in diesem Zusammenhang ist die Erschließung neuer Politikfelder. Insbesondere die Kommission ist hierbei in einer vergleichsweise starken Position, da sie formal den Prozeß der europäischen Politikformulierung kontrolliert. Sie hat daher die Möglichkeit, durch geschicktes Taktieren im Rahmen ihrer Rolle als Prozeßmanager über innovative PolicyVorschläge neue Handlungsfelder zu eröffnen. Die Formulierung der europäischen Umweltpolitik wird somit von supranationalen Institutionen beeinflußt, die aus eigenem Interesse an einer strengen und umfassenden Regulierung interessiert sind (5).

Ein weiterer Aspekt, der Policy-Innovation und strengere Regulierung begünstigt, ist der regulative Wettbewerb zwischen den Mitgliedsstaaten. Regulativer Wettbewerb entsteht aufgrund der Tatsache, daß es der europäische Entscheidungsprozeß erlaubt, Maßnahmen auch gegen den Willen einzelner Mitgliedsstaaten durchzusetzen. Für überstimmte Länder kann dies weitreichende institutionelle Anpassungszwänge an europäische Vorgaben hervorrufen. Um diesen Problemen vorzubeugen, versuchen die Staaten tendenziell, den Prozeß der europäischen Politikgestaltung in ihrem Sinne zu beeinflussen, indem sie mit eigenen Vorschlägen an die Kommission herantreten. Die Kommission, selbst an der Ausweitung ihrer Kompetenzen interessiert, ist generell sehr offen für solche Vorschläge.

Der regulative Wettbewerb zwischen den Mitgliedsstaaten verhindert auf diese Weise, daß die Umweltpolitik der EU sich als ,race to the bottom" entwickelt, denn nur innovative Vorschläge der Mitgliedsstaaten werden von der Kommission auf die Agenda gesetzt. Überdies begünstigt der regulative Wettbewerb den Patchwork-Charakter europäischer Umweltpolitik, denn nicht immer bringt der regulative Wettbewerb den gleichen Gewinner (6).

Ein zweiter kooperationsbegünstigender Effekt ergibt sich aus der Mitgliederstruktur der Gemeinschaft. Aufgrund einer vergleichsweise starken Konzentration wirtschaftlicher Stärke auf einige wenige Mitgliedsstaaten ist es in vielen Fällen ausreichend, wenn sich die ,großen" Staaten einigen. Kleinere oder wirtschaftlich weniger einflußreiche Länder spielen in diesem Prozeß meist keine dominante Rolle, sondern fungieren als Koalitionspartner der "Großen“. Auf diese Weise reduziert sich das Problem der Heterogenität nationaler Interessen und damit die Schwierigkeit, zu kooperativen Lösungen zu kommen (7). Überdies bietet der gemeinschaftliche Handlungskontext die Möglichkeit des Interessenausgleichs zwischen wirtschaftlich stärker und schwächer entwickelten Staaten im Wege von Kompensations- und Paketlösungen. Zusammenfassend läßt sich somit festhalten: Die Regulierung der europäischen Umweltpolitik auf einem relativ hohen Niveau wird durch ökonomische und politische Mechanismen begïnstigt, die es einflußreichen und umweltpolitisch ambitionierten Mitgliedsstaaten potentiell erlauben, ihre Standards auch für andere Länder verbindlich zu machen.

\section{- Vergleich mit der globalen Ebene}

Vergleicht man die umweltpolitischen Entwicklungsfaktoren, wie sie auf europäischer Ebene gegeben sind, mit den entsprechenden Voraussetzungen auf globaler Ebene, so fallen einige Unterschiede auf, die sich weniger auf die Rolle 
ökonomischer Mechanismen beziehen, sondern hauptsächlich die Möglichkeiten politischer Kooperation betreffen.

Bezüglich der ökonomischen Mechanismen scheinen die Voraussetzungen für eine regulierungsverstärkende Dynamik in ähnlicher Weise wie im europäischen Kontext gegeben zu sein. So spricht nichts dagegen, daß nicht auch auf globaler Ebene umweltpolitisch ambitionierte Staaten versuchen, durch eine Anhebung von Umweltstandards nationale Märkte zu schützen. Auf der anderen Seite ist es genauso denkbar, daß von "grïnen" Staaten ein Pull-Effekt auf Staaten ausgeht, die ihre Güter dorthin exportieren wollen. Diese Dynamik ist für sich allein jedoch nicht ausreichend, um die Herausbildung und Fortentwicklung globaler umweltpolitischer Strategien zu begünstigen.

Verglichen mit den Bedingungen supranationaler Kooperation sind die Möglichkeiten für effektive Problemlösungen durch internationale Umweltvereinbarungen jedoch grundsätzlich eingeschränkt:

Auf internationaler Ebene existieren erstens keine Institutionen, denen von den Nationalstaaten umweltpolitische Handlungskompetenzen übertragen wurden. Es gibt daher keinen globalen, mit der Rolle der EU-Kommission vergleichbaren Prozeßmanager, der innovative Regulierungen vorantreibt. Die einzige internationale Institution, die potentiell in der Lage wäre, einen Ausgleich zwischen ökonomischer Liberalisierung und Umweltregulierung herzustellen, die Welthandelsorganisation (WTO), ist - verglichen zur EU - eine schwächere und in ihrem Zuständigkeitsbereich enger definierte Organisation. Entscheidungen basieren auf der Zustimmung aller Mitglieder. Eine Dynamik im Sinne eines regulativen Wettbewerbs, bei dem einzelne Staaten auch gegen ihren Willen bestimmte Vorschläge akzeptieren müssen, ist daher nur schwer vorstellbar. Auf der anderen Seite sinkt bei dem fehlenden Risiko, durch Entscheidungen einer globalen Regulierungsinstanz einem entsprechenden institutionellen Anpassungsdruck ausgesetzt zu sein, gleichzeitig der Anreiz, auf globaler Ebene eine Vorreiterrolle zu spielen.

Ein zweites Problem globaler Umweltvereinbarungen ist der - verglichen zur EU - größere Teilnehmerkreis und die damit verbundene Heterogenität von Interessen, die sich kooperationshemmend auswirkt. Im Gegensatz zur EU bestehen weitaus größere Differenzen beziig- lich des ökonomischen Entwicklungsstandes sowie administrativer Kapazitäten unterschiedlicher Staaten, wobei die grundlegende Konfliktlinie in diesem Kontext zwischen Industrie- und Entwicklungsländern verläuft. Es bestehen keine Entscheidungs- und Kompensationsmechanismen, die es einzelnen - wirtschaftlich einflußreichen - Staaten ermöglichen, umweltpolitisch ,vorzupreschen“ und damit die anderen zum Nachziehen zu zwingen.

Natiirlich bedeutet dies nicht, daß internationale Umweltabkommen grundsätzlich erfolglos und ineffektiv sein müssen. Vielmehr zeigt sich ein relativ gemischtes Bild, wenn man die Effekte der derzeit etwa 120 bestehenden Vereinbarungen analysiert (8). Erfolgreiche Kooperationen zeigen sich aber meist nur in solchen Fällen, in denen der Teilnehmerkreis durch eine relativ homogene Interessenstruktur gekennzeichnet war; d.h. wenn es sich um Vereinbarungen zwischen „reichen“ Staaten gehandelt hat, wenig Industriezweige betroffen waren und diejenigen Staaten, die mit den größten Kosten konfrontiert wurden, gleichzeitig auch am stärksten von verbesserten Umweltregulierungen profitiert haben. Umgekehrt zeigen sich Probleme vor allem dann, wenn die effektive Bewältigung von Umweltproblemen entscheidend auf entsprechende Maßnahmen in industriell weniger entwickelten Ländern angewiesen ist (9).

\section{Erfolgsaussichten globaler Umwelt-Governance}

Betrachtet man insgesamt die Entwicklungsbedingungen für eine effektive Umweltpolitik auf globaler Ebene, so zeigt sich ein ambivalentes Bild. Einerseits, so scheint es, dürfte das eingangs erwähnte Negativszenario eines umweltpolitischen Dumpings eher unrealistisch sein, betrachtet man das Potential der ökologischen Dynamik liberaler Märkte und die - wenngleich eingeschränkten - Möglichkeiten internationaler Kooperationen. Genauso unwahrscheinlich wie ein "race to the bottom" ist jedoch auch das Positivszenario eines, ,race to the top“. Dies liegt nicht nur an den Schranken ökologischer Marktdynamiken, sondern vor allem an der Schwierigkeit, eine Vielzahl von heterogenen nationalen Interessen im Rahmen internationaler Vereinbarungen effektiv zu koordinieren. Das grundlegende Problem globaler Umweltpolitik liegt hierbei nicht darin, daß Marktliberalisierung umweltpolitisch ambitionierte Staaten davon abhält, ihre Umweltstandards $\mathrm{zu}$ ver- schärfen (sei es im Wege nationaler Alleingänge oder im Rahmen von regionalen Kooperationen, etwa im europäischen Kontext). Vielmehr besteht die Schwierigkeit darin, daß es an wirksamen, mit der europäischen Ebene vergleichbaren, globalen Mechanismen fehlt, die es ambitionierten Staaten ermöglichen, andere Länder dazu zu bewegen, ihre Umweltregulierungen zu verbessern. Wie Vogel feststellt, ,the problem is not that poor countries are driving rich country standards downward; it is rather that rich countries lack adequate mechanisms to drive poor country standards sufficiently upward" (10). Insoweit sind wir mit einem Defizit globaler Umwelt-Governance konfrontiert.

\section{Anmerkungen}

(1) Haigh, Nigel: The Manual of Environmental Policy: the EC and Britain. Catermill Publishing, London 1996.

(2) Knill, Christoph: The Impact of National Administrative Traditions on the Implementation of EU Environmental Policy. In: Journal of Public Policy, Vol. 18 (1998), №. 1. (3) Scheuing, Dieter: Umweltschutz auf der Grundlage der Einheitlichen Europäischen Akte. In: Europarecht, Nr. 2 (1989), S.152-192.

(4) Vogel, David: Trading Up. Consumer and Environmental Regulation in the Global Economy. Harvard University Press, Cambridge 1995.

(5) Knill, Christoph: Patterns of European Policy Development: The Case of Environmental Policy. In: Environmental Policy and Law, Vol. 27 (1997), No. 1, S.48-56. (6) Hèritier, Adrienne, Christoph Knill, Susanne Mingers: Ringing the Changes in Europe. Regulatory Competition and the Transformation of the State. De Gruyter, Berlin 1996.

(7) Genschel, Philipp, Thomas Plümper: Regulatory Competition and International Co-operation. In: Journal of European Public Policy, Vol. 4 (1997), No. 4, S.626-642.

(8) Haas, Peter M., Robert 0. Koehane, Marc A. Levy (Hg.): Institutions for the Earth. Sources of Effective International Environmental Protection. MIT Press, Cambridge 1993.

(9) Jacobson, Harold K., Edith Brown Weiss ( $\mathrm{Hg}$.): Engaging Countries: Strengthening Compliance with International Environmental Accords. MIT Press, Cambridge 1997. (10) Vogel, David: Trading Up and Governing Across: Transnational Governance and Environmental Protection. In: Journal of European Public Policy, Vol. 4 (1997), №. 4, S.556-571 (hier S.568).

\section{Der Autor}

Dr. Christoph Knill ist wissenschaftlicher Mitarbeiter in der Max-Planck Projekigruppe Recht der Gemeinschaftsgüter.

Kontakt: Poppelsdorferallee 45, 53115 Bonn,

Telefon: 0228 /91416-0 
(c) 20I0 Authors; licensee IÖW and oekom verlag. This is an article distributed under the terms of the Creative Commons Attribution Non-Commercial No Derivates License (http://creativecommons.org/licenses/by-nc-nd/3.o/), which permits unrestricted use, distribution, and reproduction in any medium, provided the original work is properly cited. 